\title{
Krylov Subspaces from Bilinear Representations of Nonlinear systems
}

\author{
M. Condon and R. Ivanov
}

School of Electronic Engineering, Dublin City University, Dublin 9, IRELAND

\begin{abstract}
-
For efficient simulation of state-of-the-art dynamical systems as arise in all aspects of engineering, the development of reduced-order models is of paramount importance. While linear reduction techniques have received considerable study, increasingly nonlinear model reduction is becoming a significant field of interest. From a circuits and systems viewpoint, systems involving micromachined devices or systems involving mixed technologies necessitate the development of reduced-order nonlinear models. From a control systems viewpoint, the design of controllers for nonlinear systems is greatly facilitated by nonlinear model reduction strategies. To this end, the paper proposes two novel model-reduction strategies for nonlinear systems. The first involves the development, in a novel manner as compared to previous approaches, of a reduced-order model from a bilinear representation of the system while the second involves a reducing a polynomial approximation using subspaces derived from a related bilinear representation. Both techniques are shown to be effective through the evidence of a standard test example.
\end{abstract}




\section{INTRODUCTION}

With the growing complexity and dimensionality of state-of-the-art dynamical systems as arise in all aspects of engineering, model reduction is becoming a vital aspect of modern system simulation. While model reduction techniques for linear systems are well studied (e.g. [1-5] and references therein) especially in the context of interconnect and package modelling, the study of nonlinear model reduction strategies has received considerably less attention. However, from a circuits and systems viewpoint, systems involving micromachined devices or systems involving mixed technologies necessitate the development of reduced-order nonlinear models. From a control systems viewpoint, the design of controllers for nonlinear systems is greatly facilitated by nonlinear model reduction strategies. Applications for effective nonlinear control design abound in engineering from the control of chemical process systems to the control of aeronautical and electrical power systems. Hence, the development of model reduction methods for nonlinear systems is of paramount importance to the general engineering community. Unfortunately, the study of nonlinear systems is much more complicated since their solutions (when they exist) can be of a quite complex nature (not unique, singular, chaotic etc.). Therefore, the development of suitable reduced-order modelling techniques represents a formidable challenge. Some recent work in this field is presented in [6-15].

The present contribution proposes two novel techniques involving Krylov subspaces for model reduction of weakly nonlinear systems. The particular choice of Krylov subspace model reduction stems from the success of the Krylov paradigm in linear model reduction. Firstly for linear systems, the choice of a projection matrix is 
straightforward resulting from the moment-matching properties of the transfer function of the system. Secondly, the computation the projection matrix is straightforward involving only the solution of linear equations or matrix products. Furthermore, the Krylov approach enables the efficient formation and simulation of a reduced order model in that the reduced order model has the same form as the original system but is of much lower dimensionality. However, the development of Krylov approaches for nonlinear model reduction is not quite so straightforward. Consider the following nonlinear system:

$$
\begin{aligned}
& \dot{x}(t)=f(x(t))+B u(t) \\
& y(t)=C x(t)
\end{aligned}
$$

where $f: \Re^{n} \rightarrow \Re^{n}$ is a non-linear function, with the initial condition $x(0)=x_{0}$ and $u(t), y(t) \in \mathfrak{R}^{1} . B, C \in \mathfrak{R}^{n}$ are constant vectors ( $C$ is a vector-row and $B$ is a vectorcolumn). To directly translate the projection scheme developed for linear model reduction would involve the determination of a projection matrix $V$ which is orthogonal, $V^{T} V=I$, such that

$$
\begin{aligned}
& \dot{x}_{r}(t)=V^{T} f\left(V x_{r}(t)\right)+\hat{B} u(t) \\
& \hat{y}(t)=C V x_{r}(t)
\end{aligned}
$$

where $x(t)=V x_{r}(t)$ and $x_{r}(t)$ is the reduced state space. $\hat{B}=V^{T} B$ and the aim is that $\hat{y} \approx y$ where $\hat{y}$ is the output of the reduced model. However, to date, there has been no universal approach proposed for the determination of $V$. Furthermore, as outlined in [12], the interpretation of (2) as a reduced-order model for a nonlinear system is dubious. Since $f$ is a nonlinear function, it is not, in general, possible to pass $V$ through the parentheses in (2) and thus computation of the nonlinear function, $f$, is unavoidable. Since the computation of such a function is often the major determining factor in the overall system simulation time, a reduction in the size of the state-space 
if achieved in this manner may not produce the desired effect as regards a significant reduction in computation time.

Thus, for nonlinear systems, some compromises have to be made if Krylov approaches are to be utilised. For this contribution, the compromise comprises either a restriction on the type of nonlinear system under consideration or an approximation of the nonlinear equations describing the system behaviour. In particular, two categories of nonlinear system representation will be considered - bilinear system representations and polynomial system representations. A bilinear system is one which is linear in state, linear in control but not linear jointly. Bilinear systems frequently arise naturally in engineering, for example, nuclear fission, chemical and biological models and ecological models [17]. However, even when the system itself is not naturally bilinear, the bilinear representation offers a superior representation to a linear model. Polynomial representations also aim to improve on a basic linear model by incorporating in the system representation the higher-order terms in a series expansion of the nonlinear function, $f$.

While some Krylov subspace based approaches have been proposed for nonlinear model reduction eg [10-12] employing bilinear and polynomial representations, the current work employs them in a rather different manner to that previously presented. Full details of the new approaches and their position relative to existing methods will be detailed in subsequent sections. Section 2 will present the first approach and the second is detailed in Section 3. An illustrative and standard example [10-15] is given in Section 4, which confirms the efficacy of the proposed approaches. It should be noted that this example is not intended to be a practical application of the techniques. 
It is chosen to enable ease of comparison of the proposed techniques with existing approximations and to confirm the theoretical proposals put forth throughout the paper.

\section{BILINEAR APPROXIMATION OF WEAKLY NONLINEAR SYSTEMS}

Consider again the nonlinear system in (1). For the ensuing analysis, it is assumed that the system (1) is weakly nonlinear with an asymptotically stable equilibrium point as described in [10]. Without loss of generality, it is assumed that $x=0$ is the stable equilibrium point of the system i.e. $f(0)=0$. Under this assumption $f(x)$ can be expanded in a generalised Taylor's series about the equilibrium point $x=0$ :

$$
f(x)=A_{1} x^{(1)}+A_{2} x^{(2)}+A_{3} x^{(3)}+\ldots
$$

where $x^{(1)}=x, x^{(2)}=x \otimes x, \quad x^{(3)}=x \otimes x \otimes x$, etc. and $\otimes$ denotes the Kronecker product. Stability of the system implies that all the eigenvalues of $A_{1}$ have negative real parts.

The systems under consideration will be assumed to be weakly nonlinear. Hence, the condition that each term in the Taylor's expansion is small compared to the previous one will be taken to hold. Consequently, the system in (1) can be approximated by the well-known bilinear representation (Carleman bilinearization) of (1) [10]:

$$
\begin{aligned}
\dot{\hat{x}}(t) & =\hat{A} \hat{x}(t)+\hat{N} \hat{x}(t) u(t)+\hat{B} u(t) \\
y(t) & =\hat{C} \hat{x}(t)
\end{aligned}
$$

where

$$
\hat{x}(t)=\left[\begin{array}{c}
x^{(1)} \\
x^{(2)} \\
\vdots
\end{array}\right]
$$


and $\hat{A}, \hat{N}, \hat{B}$ and $\hat{C}$ are constant matrices:

$$
\hat{A}=\left[\begin{array}{cccc}
A_{1} & A_{2} & \ldots & \\
& A_{21} & A_{22} & \\
& & A_{31} & A_{32} \\
& & & \ddots
\end{array}\right] \hat{N}=\left[\begin{array}{cccc}
0 & 0 & \cdots & \\
B_{20} & 0 & 0 & \cdots \\
& B_{30} & 0 & \\
& & & \ddots
\end{array}\right] \quad \hat{B}=\left[\begin{array}{c}
B \\
0 \\
\vdots
\end{array}\right] \hat{C}=\left[\begin{array}{lll}
C & 0 & \cdots
\end{array}\right] .
$$

The matrices $A_{i}$ are defined from the Taylor's series expansion in (3) and

$$
\begin{aligned}
& A_{j i}=A_{i} \otimes I \otimes \cdots \otimes I+I \otimes A_{i} \otimes \ldots \otimes I+\cdots+I \otimes I \otimes \cdots \otimes A_{i}, \\
& B_{j 0}=B \otimes I \otimes \cdots \otimes I+I \otimes B \otimes \ldots \otimes I+\cdots+I \otimes I \otimes \cdots \otimes B
\end{aligned}
$$

where $I$ is the $n \times n$ identity matrix and there are $j$ terms in each sum. Thus $\hat{A}$, $\hat{N}$ are square matrices of dimension $n+n^{2}+\cdots n^{K} ; \hat{x}, \hat{B}, \hat{C}$ are vectors with $n+n^{2}+\cdots n^{K}$ components if $K$ terms in the Taylor's series expansion are taken into account.

As stated in the introduction, the rationale for employing the bilinear representation is that it allows higher-order terms to be explicitly incorporated in the subsequent model reduction technique and hence is superior to employing a linear representation. However, for practicality purposes, the matrix $\hat{x}(t)$ requires truncation. For the present work, $\hat{x}$ is taken as:

$$
\hat{x}(t)=\left[\begin{array}{l}
x^{(1)} \\
x^{(2)}
\end{array}\right]
$$

which corresponds to taking into account the quadratic terms in (3).

Consequently,

$$
\hat{A}=\left[\begin{array}{cc}
A_{1} & A_{2} \\
0 & A_{21}
\end{array}\right], \quad \hat{N}=\left[\begin{array}{cc}
0 & 0 \\
N & 0
\end{array}\right], \quad \hat{B}=\left[\begin{array}{c}
B \\
0
\end{array}\right], \quad \hat{C}=\left[\begin{array}{ll}
C & 0
\end{array}\right]
$$


where $A_{1}$ and $A_{2}$ are the matrices in (3), $A_{21}=A_{1} \otimes I+I \otimes A_{1}, N=B \otimes I+I \otimes B$ where $I$ is the $n \times n$ identity matrix. Thus, $\hat{A}$ and $\hat{N}$ are square matrices of dimension $n+n^{2} ; \hat{x}, \hat{B}, \hat{C}$ are vectors with $n+n^{2}$ components.

Now for the purposes of developing the new Krylov approach, consider, initially, the case of a bilinear system subject to a constant input $\bar{u}$.

$$
\begin{aligned}
\dot{\hat{x}}(t) & =(\hat{A}+\bar{u} \hat{N}) \hat{x}(t)+\hat{B} \bar{u} \\
y(t) & =\hat{C} \hat{x}(t)
\end{aligned}
$$

This obviously results in the linear system (8) and thus the system, (8), possesses all the advantageous properties pertaining to linear systems. Bearing this observation in mind, for the general case where $u(t) \neq c o n s t$, it is proposed to introduce a parameter $\kappa$ which is related to $u(t): \kappa=\kappa[u] \quad(\kappa[u]=\bar{u}$ for constant input $)$ and $-K_{B} \leq \kappa[u] \leq K_{B}$ reflecting the fact that $|u(t)| \leq K_{B}$ where $K_{B}$ is a constant bound. It is assumed that $\kappa$ is a functional on $u$ (i.e. a parameter that does not depend on $t$ but does depend on the behaviour of $u(t)$ for $t \in[0, \tau]$ and on the specific choice of interval, $[0, \tau])$. The dependence of $\kappa$ on $u(t)$ is important. This enables the Krylov subspaces of the system (8) to vary with $u(t)$ which results in a superior model reduction technique for a bilinear representation. Hence, in order to define $\kappa[u]$, consider a rescaling of the input $u \rightarrow \mu$ where $\gamma$ is a constant and $\mu$ is sufficiently small. This transforms the bilinear system into another bilinear system with source $u(t)$ as follows:

$$
\begin{aligned}
& \dot{\hat{x}}(t)=\hat{A} \hat{x}(t)+\hat{\mathcal{N}} \hat{x}(t) u(t)+(\gamma \hat{B}) u(t) \\
& y(t)=\hat{C} \hat{x}(t)
\end{aligned}
$$

A rescaling of $\hat{B}$ does not affect the determination of Krylov spaces. 
However, the term $\hat{N} \hat{N} \hat{x} u$ in (9) shows that in order to determine the (input-dependent) Krylov subspaces it is necessary to impose the validity of the following property of $\kappa:$

$$
\kappa[\mu]=\gamma \kappa[u]
$$

i.e. $\kappa[u]$ must be a linear functional on $u$. Therefore, for the general case of a nonconstant input, it is reasonable to define $\kappa[u]$ as follows:

$$
\kappa[u]=\frac{1}{\tau} \int_{0}^{\tau} u(t) d t
$$

The definition in (11) obviously obeys (10).

Of course, for control systems, where the input controls the output, $u(t)$ is not given $a$ priori but nevertheless (11) provides an estimate on how the parameter $\kappa$ relates to the typical values of the input. In general, the best value of $\kappa$ for a particular control system can be found from computer simulations.

The proposition then is to employ the following linear system that is related to the original bilinear system to extract a projection subspace for the bilinear system with a $\kappa[u]$ value determined from (11) or by some other means:

$$
\begin{aligned}
\dot{\hat{x}}(t) & =(\hat{A}+\kappa \hat{N}) \hat{x}(t)+\hat{B} u(t) \\
y(t) & =\hat{C} \hat{x}(t)
\end{aligned}
$$

When the system in (12) is represented in the frequency domain, the input and output are related by the following transfer function:

$$
H(s)=\hat{C}\left(s \hat{I}-\hat{A}_{\kappa}\right)^{-1} \hat{B}
$$


where $\hat{A}_{\kappa}=\hat{A}+\kappa \hat{N} \quad$ and $\hat{I}$ is the corresponding $n+n^{2}$ dimensional identity matrix.

$H(s)$ may be expanded about a selected expansion point $s_{0}$ as follows:

$$
\begin{aligned}
& H(s)=\hat{C}\left(\left(s-s_{0}\right) \hat{I}-\left(\hat{A}_{\kappa}-s_{0} \hat{I}\right)\right)^{-1} \hat{B} \equiv \hat{C}\left(\left(s-s_{0}\right) \hat{I}-\hat{A}_{\kappa, s_{0}}\right)^{-1} \hat{B}= \\
& =\sum_{p=0}^{\infty} m_{p}\left(s-s_{0}\right)^{p}
\end{aligned}
$$

where

$$
\hat{A}_{\kappa, s_{0}} \equiv \hat{A}_{\kappa}-s_{o} \hat{I}=\hat{A}+\kappa \hat{N}-s_{0} \hat{I}
$$

and $m_{p}$ (termed moments) are:

$$
m_{p}=-\hat{C} \hat{A}_{\kappa, s_{0}}^{-(p+1)} \hat{B}
$$

If $H(s)$ is to be computed in an efficient manner using a truncated version of the summation expression in (14) then the sequence of moments $\left\{m_{p}\right\}$ must decrease rapidly in absolute value to zero with increasing $p$. This is clearly dependent on the choice of expansion point $s_{0}$. For example, if the absolute value of all eigenvalues of $\hat{A}_{\kappa}$ is greater than 1 , then the absolute values of all eigenvalues of $\hat{A}_{\kappa}^{-1}$ are less than 1 and $\left\{m_{p}\right\}$ decreases with $p$ for $s_{0}=0$. In such a case $s_{0}=0$ is a suitable expansion point for moment matching. Otherwise, (e.g. when $\hat{A}_{\kappa}$ has small eigenvalues), an appropriate expansion point should be chosen such that the absolute value of all eigenvalues of $\hat{A}_{\kappa, s_{0}}$ is greater than 1 .

For model reduction purposes, what is of interest is the relationship between the moments and the formation of suitable projection subspaces. All of the moments (16) 
may be written as scalar products between the following left and right Krylov spaces e.g. [10]:

$$
\begin{aligned}
& \hat{K}_{R 1}\left(\hat{A}_{\kappa, s_{0}}^{-1}, \hat{B}\right) \equiv \operatorname{span}\left\{\hat{B}, \hat{A}_{\kappa, s_{0}}^{-1} \hat{B}, \ldots, \hat{A}_{\kappa, s_{0}}^{\left(n+n^{2}-1\right)} \hat{B}\right\}, \\
& \hat{K}_{L 1}\left(\hat{C}, \hat{A}_{\kappa, s_{0}}^{-1}\right) \equiv \operatorname{span}\left\{\hat{C}, \hat{C} \hat{A}_{\kappa, s_{0}}^{-1}, \ldots, \hat{C} \hat{A}_{\kappa, s_{0}}^{-\left(n+n^{2}-1\right)}\right\} .
\end{aligned}
$$

(The notation is adopted throughout that Krylov subspaces of the form $\hat{K}$ have dimension $n+n^{2} \ldots$ while Krylov spaces of the form $K$ have dimension $n$ ).

The projection subspace is formed by taking the first $k$ vectors of each Krylov space. To avoid ill-conditioning in the reduction matrices, the two bases can be made biorthogonal, i.e. $W V=I_{k}$ where the vector-columns of $V$ are from $\hat{K}_{R 1}$, the vectorrows of $W$ are from $\hat{K}_{L 1}$ and $I_{k}$ is the $k \times k$ identity matrix.

The state vector $\hat{x}(t)$ may then be approximated by the 'reduced' $k$-dimensional state vector $x_{r}(t)$ i.e. $\hat{x}(t) \approx V x_{r}(t)$. The resultant reduced bilinear system is therefore:

$$
\begin{aligned}
& \dot{x}_{r}(t)=A_{r} x_{r}(t)+N_{r} x_{r}(t) u(t)+B_{r} u(t) \\
& y(t)=C_{r} x_{r}(t)
\end{aligned}
$$

where $A_{r}=W \hat{A} V, N_{r}=W \hat{N} V, B_{r}=W \hat{B}$ and $C_{r}=\hat{C} V$.

Thus, by employing the system in (12) it is possible to define suitable Krylov spaces that may subsequently be used for determining a reduced-order bilinear system. The success of employing such subspaces will be evident from the results in Section 4 .

\section{POLYNOMIAL APPROXIMATION OF WEAKLY NONLINEAR SYSTEMS}

The second technique proposed for model reduction of weakly nonlinear systems is based on a polynomial approximation of the given system (1). However, for ease of explanation we will restrict ourselves in the current work to a quadratic approximation which is as follows: 


$$
\begin{aligned}
& \dot{x}(t)=A_{1} x+A_{2} x \otimes x+B u(t) \\
& y(t)=C x(t)
\end{aligned}
$$

Particular observations in relation to a corresponding bilinear approximation of the nonlinear system can provide some insight into the construction of a suitable Krylov space for model reduction of the quadratic representation. Therefore, the first part of this section will again focus on the bilinear representation. Consider the solution to the bilinear system in (4) [16]:

$$
\hat{y}(t)=y_{x}(t)+y_{u}(t)+y_{x u}(t)
$$

where $y_{x}(t)=\hat{C} e^{\hat{A} t} \hat{x}_{0}$,

$$
\begin{aligned}
& y_{u}(t)=\sum_{i=1}^{\infty} \frac{1}{i !} \int_{0}^{t} \ldots \int_{0}^{t} w_{i}\left(t_{1}, \ldots, t_{i}\right)\left[\prod_{k=1}^{i} u\left(t-t_{k}\right)\right] d t_{1} \ldots d t_{i} \\
& y_{x u}(t)=\sum_{i=1}^{\infty} \frac{1}{i !} \int_{0}^{t} \ldots \int_{0}^{t} z_{i}\left(t_{1}, \ldots, t_{i}\right) \hat{N} e^{\hat{A}\left(t-t_{1}\right)} \hat{x}_{0}\left[\prod_{k=1}^{i} u\left(t-t_{k}\right)\right] d t_{1} \ldots d t_{i} \\
& w_{i}\left(t_{1}, \ldots, t_{i}\right)=\sum_{\wp_{i}} v_{i}\left(t_{1}, \ldots, t_{i}\right) \\
& v_{1}\left(t_{1}\right)=\hat{C} e^{\hat{A} t_{1}} \hat{B} \\
& v_{i}\left(t_{1}, \ldots, t_{i}\right)=\hat{C} e^{\hat{A} t_{i}} \hat{N} e^{\hat{A}\left(t_{i-1}-t_{i}\right)} \ldots \hat{N} e^{\hat{A}\left(t_{1}-t_{2}\right)} \hat{B}\left[\prod_{k=0}^{i-2} \theta\left(t_{k+1}-t_{k+2}\right)\right],
\end{aligned}
$$

$\wp_{i}$ is the set of permutations of $\left\{t_{1}, \ldots, t_{i}\right\}$ and $\theta$ is the unit step function. The expression for $z_{i}$ can be obtained from those for $v_{i}$ with $\hat{B}$ replaced by the identity matrix $I$.

The kernels $\hat{C} e^{\hat{A} t_{i}} \hat{N} e^{\hat{A}\left(t_{i-1}-t_{i}\right)} \ldots \hat{N} e^{\hat{A}\left(t_{1}-t_{2}\right)} \hat{B}$ of this solution naturally lead to the multidimensional transfer functions of the form [10]:

$$
H_{i}\left(s_{1}, s_{2}, \ldots, s_{i}\right)=\hat{C}\left(s_{i} \hat{I}-\hat{A}\right)^{-1} \hat{N} \ldots \hat{N}\left(s_{1} \hat{I}-\hat{A}\right)^{-1} \hat{B}
$$


The coefficients in a power series expansion of $H_{i}$ (about $s_{0}$ ) are the following multi-moments:

$$
m_{i}\left(l_{1}, l_{2}, \ldots, l_{i}\right)=(-1)^{i} \hat{C} \hat{A}_{s_{0}}^{-l_{i}} \hat{N} \ldots \hat{N} \hat{A}_{s_{0}}^{-l_{2}} \hat{N} \hat{A}_{s_{0}}^{-l_{1}} \hat{B}
$$

where $l_{i}$ are nonnegative integers and $\hat{A}_{s_{0}}=\hat{A}-s_{0} \hat{I}$.

The matrices involved in (22) have the following noteworthy structure:

$$
\begin{aligned}
& \hat{A}_{s_{0}}^{-1}=\left[\begin{array}{cc}
A_{1, s_{0}}^{-1} & -A_{1, s_{0}}^{-1} A_{2} A_{21, s_{0}}^{-1} \\
0 & A_{21, s_{0}}^{-1}
\end{array}\right] \\
& \hat{A}_{s_{0}}^{-l}=\left[\begin{array}{cc}
A_{1, s_{0}}^{-l} & -\sum_{l_{1}+l_{2}=l} A_{1, s_{0}}^{-l_{1}} A_{2} A_{21, s_{0}}^{-l_{2}} \\
0 & A_{21, s_{0}}^{-l}
\end{array}\right]
\end{aligned}
$$

where $A_{1, s_{0}}=A_{1}-s_{0} I$ and $A_{21, s_{0}}=A_{21}-s_{0} I \otimes I$.

As a result of this structure and that of $\hat{B}$ and $\hat{C}$ (see Section 2 (7)), the kernels of degree 1 may be written as:

$$
m_{1}(l)=-\hat{C} \hat{A}_{s_{0}}^{-l} \hat{B}=-C A_{1, s_{0}}^{-l} B
$$

This indicates that they can therefore be represented as scalar products between vectors of the following $n$-dimensional (not $n+n^{2}$-dimensional) Krylov spaces:

$$
\begin{aligned}
& K_{R, 1}\left(A_{1, s_{0}}^{-1}, B\right) \equiv \operatorname{span}\left\{B, A_{1, s_{0}}^{-1} B, \ldots, A_{1, s_{0}}^{-(n-1)} B\right\}, \\
& K_{L, 1}\left(C, A_{1, s_{0}}^{-1}\right) \equiv \operatorname{span}\left\{C, C A_{1, s_{0}}^{-1}, \ldots, C A_{1, s_{0}}^{-(n-1)}\right\} .
\end{aligned}
$$

Similarly, kernels of degree 2 may also be represented as scalar products between vectors of $n$-dimensional Krylov spaces:

$$
m_{2}(p, l)=\hat{C} \hat{A}_{s_{0}}^{-l} \hat{N} \hat{A}_{s_{0}}^{-p} \hat{B}=-\sum_{l_{1}+l_{2}=l} C A_{1, s_{0}}^{-l_{1}} A_{2} A_{21, s_{0}}^{-l_{2}} N A_{1, s_{0}}^{-p} B
$$

The combined Krylov spaces are as follows:

$$
\begin{aligned}
& K_{R, 2}=K_{R, 1}\left(A_{1, s_{0}}^{-1}, A_{2} \cdot K_{R, 1}\left(A_{21, s_{0}}^{-1}, N \cdot K_{R, 1}\left(A_{1, s_{0}}^{-1}, B\right)\right) \equiv \bigcup_{l_{1}, l_{2} \geq 0, p>0} \operatorname{span}\left\{A_{1, s_{0}}^{-p} B ; A_{1, s_{0}}^{-l_{1}} A_{2} A_{21, s_{0}}^{-l_{2}} N A_{1, s_{0}}^{-p} B\right\},\right. \\
& K_{L, 2}=K_{L, 1}\left(K_{L, 1}\left(K_{L, 1}\left(C, A_{1, s_{0}}^{-1}\right) \cdot A_{2}, A_{21, s_{0}}\right) \cdot N, A_{1, s_{0}}^{-1}\right) \equiv \bigcup_{l_{1}, l_{2} \geq 0, p>0} \operatorname{span}\left\{C A_{1, s_{0}}^{-l_{1}} ; C A_{1, s_{0}}^{-l_{1}} A_{2} A_{21, s_{0}}^{-l_{2}} N A_{1, s_{0}}^{-p}\right\} .
\end{aligned}
$$




$$
\begin{aligned}
& K_{R}=K_{R, 1} \cup K_{R, 2} \\
& K_{L}=K_{L, 1} \cup K_{L, 2}
\end{aligned}
$$

Thus, the crucial observation is that $\boldsymbol{n}$-dimensional Krylov spaces suffice for matching both degree 1 and degree 2 kernels (and possibly some of the degree 3 kernels) for a bilinear system.

Since the bilinear system of (4)-(7) was formed on the basis of inclusion only of quadratic terms in the Taylor's series approximation of the original system, it is proposed that the Krylov spaces in (28) can be used for reduction of the $n$ dimensional quadratic system in (19). Taking the relevant $k$-dimensional (where $k$ is the order of the reduced system) left and right subspaces, biorthogonal bases and projection matrices $V$ and $W$ can be constructed as described in Section 2. The reduced quadratic system is then:

$$
\begin{aligned}
& \dot{x}_{r}(t)=A_{1, r} x_{r}(t)+A_{2, r} x_{r} \otimes x_{r}+B_{r} u(t) \\
& y(t)=C_{r} x_{r}(t)
\end{aligned}
$$

where $A_{1, r}=W A_{1} V, A_{2, r}=W A_{2}(V \otimes V), B_{r}=W B, C_{r}=C V$

and $x(t) \approx V x_{r}(t)$.

4. AN ILLUSTRATIVE EXAMPLE

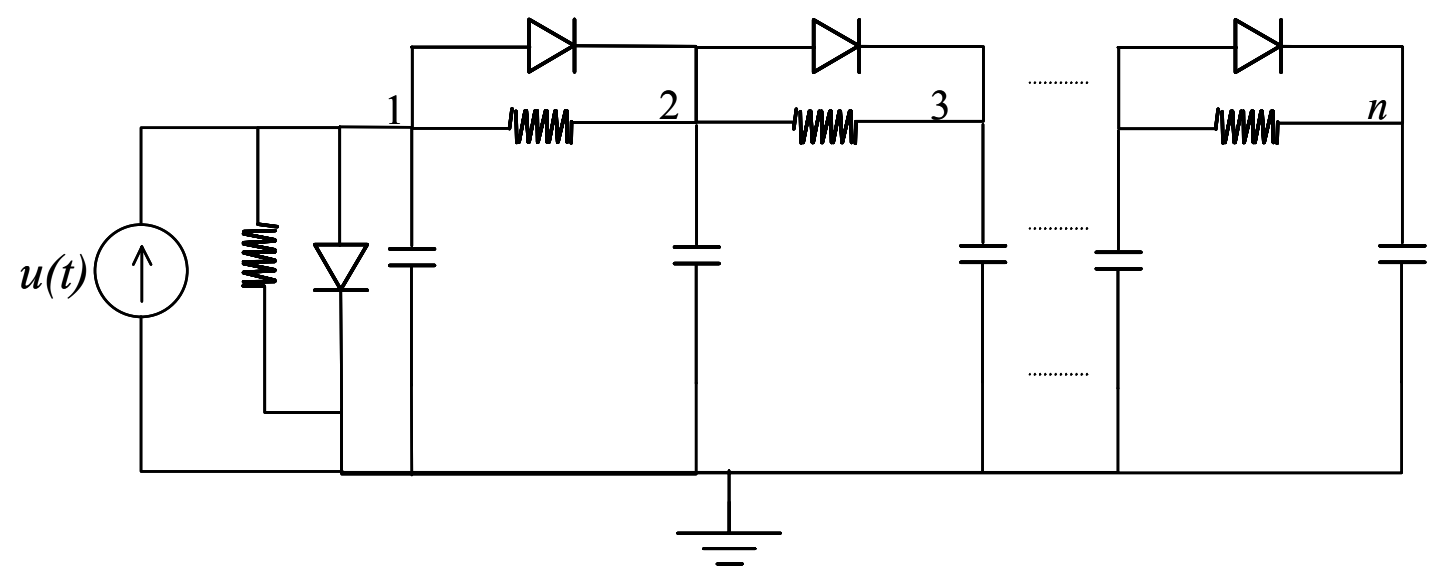

Fig 1. Nonlinear RC ladder 
The circuit employed is the nonlinear $R C$ ladder shown in Fig. 1 (frequently employed as a test circuit for model reduction techniques [10-15]). The nonlinear resistors (a diode in parallel with a unit resistor) have the constitutive relation $i(v)=\left(e^{40 v}-1\right)+v$ and the capacitors have unit capacitance.

The input to the system is a current source $u(t)=e^{-t}$ entering node 1 and the output is the voltage taken at node 1 . The number of nodes in the system is $n=30$.

The system is initially approximated by a bilinear system of order $30+30^{2}=930$. The time interval chosen for consideration is $t \in[0,1]$, i.e. $\tau=1$ and hence the parameter $\kappa$ in (12) is evaluated as $\kappa=\int_{0}^{1} e^{-t} d t=1-e^{-1}=0.6321$.

The reduction process for the bilinear system of order 930 to a bilinear system of order three is implemented utilising the Krylov spaces defined by (17) (Method 1). In order to compare various results, the root-mean squared error is calculated between the outputs of the bilinear model (4) of order 930 and the reduced-order models (18). Table 1 shows the results achieved with $\kappa=0.6321$ for a selection of expansion points $s_{0}$. (Obviously, tests were carried out for a much larger range of expansion points and what is given in Table 1 corresponds only to a suitable selection). The variation in the results clearly shows the importance of judicious choice of expansion point. The best result is achieved with and expansion point of $s_{0}=2.7$ with the corresponding rms error equal to $\mathbf{1 . 1} \times \mathbf{1 0}^{-5}$. To confirm the validity and superiority of this new approach (Method 1), results are also shown for the case where $\kappa=0$. This corresponds to the standard linear approximation of a bilinear representation. With 
$\kappa=0$ the rms error is $\sim 10^{-3}$ for a large range of values of $s_{0}$ around 3.0 (where the rms values are at their lowest). Phillips [12] also proposes determining a projection basis for bilinear systems based on a Krylov approach. He chooses an initial basis $V_{1}$ from $\hat{K}_{L 1}=\hat{K}_{R 1}=\hat{K}\left(\hat{A}^{-1}, \hat{B}\right)$ such that first-order kernels are matched up to terms in $s^{q 1}-\hat{A}^{-m} \hat{B} \in \operatorname{span}\left(V_{1}\right) \quad m<q_{1}$. He then proceeds to determine a basis $V_{2}$ from $\hat{K}_{L 2}=\hat{K}_{R 2}=\hat{K}\left(\hat{A}^{-1}, \hat{N} \hat{K}_{R 1}\left(\hat{A}^{-1}, \hat{B}\right)\right)$ and finally constructs $V \in R^{n \times k}(k$ is the order of the reduced system) from $\hat{K}_{L}=\hat{K}_{R}=\hat{K}_{L 1} \cup \hat{K}_{L 2}$. With this approach the second order regular kernels of the reduced model and the original bilinear system match up to terms in $s^{q 1} s^{q 2}-\hat{A}^{-l} \hat{N} \hat{A}^{-m} \hat{B}=V \hat{A}_{r}{ }^{-l} \hat{N}_{r} \hat{\mathrm{A}}_{r}{ }^{-m} \hat{B}_{r} \quad m<q_{1} \quad l<q_{2}$ where the subscript $r$ denotes matrices of the reduced-order model. Implementation of this approach in conjunction with biorthogonalisation [10] yields an $\mathrm{rms}$ error of $2.4 \times 10^{-4}$. Biorthogonalisation is known to be more efficient in multimoment matching than the use of one-sided Krylov subspaces [10].

As evidenced by these results, the new method proposed in this work with a suitable choice of expansion point leads to greatly improved results.

Table 1: Results for nonlinear ladder

\begin{tabular}{|c|c|c|}
\hline$\kappa$ & $S_{\text {n }}$ & Rms error \\
\hline 0 & 0 & $1.3 \times 10^{-2}$ \\
\hline 0 & $3.0 \pm 1.0$ & $(6.5 \pm 0.2) \times 10^{-3}$ \\
\hline 0.6321 & 0 & $1.7 \times 10^{-2}$ \\
\hline 0.6321 & 2.4 & $2.9 \times 10^{-4}$ \\
\hline 0.6321 & 2.5 & $1.9 \times 10^{-4}$ \\
\hline 0.6321 & 2.6 & $1.0 \times 10^{-4}$ \\
\hline $\mathbf{0 . 6 3 2 1}$ & $\mathbf{2 . 7}$ & $\mathbf{1 . 1 \times 1 0 ^ { - 5 }}$ \\
\hline 0.6321 & 2.8 & $6.4 \times 10^{-5}$ \\
\hline 0.6321 & 2.9 & $1.3 \times 10^{-4}$ \\
\hline 0.6321 & 3.0 & $2.0 \times 10^{-4}$ \\
\hline
\end{tabular}

The second approach is that suggested in Section 3 whereby the system is approximated by a quadratic system (19) of order 30. The reduction to a $k=3$ 
dimensional quadratic system is implemented by utilising the following Krylov subspaces (based on (28)):

$$
\begin{aligned}
& \operatorname{span}\{V\}=\left\{B, A_{1, s_{0}}^{-1} B, A_{1, s_{0}}^{-2} B\right\}, \\
& \operatorname{span}\left\{W^{T}\right\}=\left\{C, C A_{1, s_{0}}^{-1}, C A_{1, s_{0}}^{-1} A_{2} A_{21, s_{0}}^{-1} N A_{1, s_{0}}^{-1}\right\}^{T} .
\end{aligned}
$$

and the chosen expansion point is $s_{0}=2.3$. This particular choice of subspaces and expansion point is found to yield the best results. The subspace selection is based on matching the first four of the first-order kernels as the first-order kernels contribute most to the response of the system. The elements of $\operatorname{span}\{V\}$ are from $K_{R, 1}\left(A_{1, s_{0}}^{-1}, B\right)$ in (28). The first two elements of $\operatorname{span}\left\{W^{T}\right\}$ are selected from $K_{L, 1}\left(C, A_{1, s_{0}}^{-1}\right)$ and the third element is selected from $K_{L, 2}$. The root-mean squared error is calculated between the outputs of the quadratic model (19) and the reduced-order model (29). The rms error achieved is $1.0 \times 10^{-4}$ highlighting the efficacy of this second novel approach. If a projection basis is chosen based solely on a linear approximation of the system, the rms error is $7.6 \times 10^{-4}$. It should be noted that choosing a basis based on a linear approximation of the system is the standard approach for reducing a quadratic model [13] (and also indicated in [15]). These results clearly indicate that the proposed approach leads to significant improvements.

\section{CONCLUSIONS}

Two novel methods involving Krylov subspaces for model reduction of weakly nonlinear systems have been proposed. The first method is based on bilinearisation of the system and utilizing the Krylov basis for a related linear system (12). The second approach involves using Krylov bases identified for a bilinear representation of the system for subsequent reduction of a polynomial approximation of the system. 
The first method (that proposed in Section 2) has the advantage that the choice of Krylov bases is straightforward as is the case for all linear systems. It consists of the selecting the first $k$ vectors of the set given in (17). The drawback with the approach is that which is common to all techniques that involve working directly with bilinear systems and is that the size of bilinear system is large i.e. $n^{2}+n$ if only quadratic terms are taken into account. Another point to note is that an extra parameter is introduced into the system, $\kappa$. However, an approach is suggested for the determination of this parameter based on taking the average value of the input over the interval of interest. For an input such as $u(t)=\sin \omega t$ over a large time interval, $\tau \rightarrow \infty$, clearly the average value will be zero and the system is approximated with the linear part of the bilinear representation. However, for cases of inputs with a nonzero average over a finite interval such as a decaying exponential or a step input, then utilising (12) is advantageous. The paper also indicates the necessity of judicious choice of expansion point, $s_{0}$, in model reduction methods involving series expansions in general.

The advantage of the method proposed in Section 3 is that there is no need to work directly with the large bilinear system. All that is required is to employ the Krylov spaces resulting from a bilinear representation of the system. Furthermore, there is no new parameter introduced into the modelling process. However, once again the choice of expansion point is a factor that must be taken into account in the application of reduction technique. Also, the selection of the vectors from the general Krylov spaces (28) needs to be made judiciously. 
Both approaches have been seen to yield greatly improved results over standard related techniques.

\section{References}

[1] A.C. ANTOULAS, D.C. SORENSEN and S. GUGERCIN: 'A survey of model reduction methods for large-scale systems', Contemporary Mathematics, AMS Publications, 2001

[2] K. GALLIVAN, E. GRIMME and P. VAN DOOREN: 'Model reduction of largescale systems: rational Krylov versus balancing techniques', Error Control and Adaptivity in Scientific Computing, Eds. Bulgak \& Zenger, C-536, pp. 177-190, 1999 [3] A. ODABASIOGLU, M. CELIK and L. T. PILEGGI: 'PRIMA: Passive reduced order interconnect macromodelling algorithm', IEEE Trans. Computer-Aided Design of Integrated Circuits and Systems, 17(8): 645-654, 1998

[4] L.M. SILVEIRA, M. KAMON and J.K. WHITE: 'Efficient reduced-order modelling of frequency dependent coupling inductances associated with 3-d interconnect structures', Proc. DAC'95, San Francisco, CA, June 1995, pp. 376-380

[5] P. FELDMANN and R.W. FREUND: 'Efficient linear circuit analysis by Padé approximation via the Lanczos process', IEEE Trans. CAD, vol. 14, pp. 639-649, May 1995

[6] S. LALL, J.E. MARSDEN and S. GLAVAŠKI: 'A subspace approach to balanced truncation for model reduction of nonlinear control systems', International Journal of Robust and Nonlinear Control 2002; Vol. 12: pp. 519-535.

[7] J. HAHN and T.F. EDGAR: 'An Improved Method for Nonlinear Model Reduction Using Balancing of Empirical Gramians', Computers and Chemical Engineering 2002; 26(10): pp. 1379-1397. 
[8] V. BALAKRISHNAN, Q. SU and C.-K. KOH: 'Efficient balance and truncate model reduction for large scale systems', Proc. American Control Conf. pp. 47464751, June 2001

[9] P.K. GUNUPUDI and M.S. NAKHLA: 'Model reduction of nonlinear circuits using Krylov-space techniques', Proc. DAC99, pp. 13-16, 1999.

[10] Z. BAI and D. SKOOGH: 'Krylov subspace techniques for reduced-order modeling of nonlinear dynamical systems', Electronic Proceedings of the $15^{\text {th }}$ International Symposium on the Mathematical Theory of Networks and Systems, University of Notre Dame, South Bend, Indiana, USA, 2002

[11] J.R. PHILLIPS: 'Projection frameworks for model reduction of weakly nonlinear systems', Proc. DAC2000, LA California.

[12] J.R. PHILLIPS: 'Projection-based approaches for model reduction of weakly nonlinear, time-varying systems', IEEE Transactions on computer-aided design of integrated circuits and systems, vol. 22, no. 2, February 2003

[13] Y. CHEN and J. WHITE: 'A quadratic method for nonlinear model order reduction', Inter. conf. on modelling and simulation of Microsystems semiconductors, sensors and actuators, San Diego, 2000

[14] M. REWIEŃSKI and J. WHITE: 'A trajectory piecewise-linear approach to model order reduction and fast simulation of nonlinear circuits and micromachined devices', IEEE Transactions on Computer-aided Design of Integrated Circuits and Systems, Vol. 22, No. 2, pp. 155-170, 2003

[15] N. DONG and J. ROYCHOWDHURY: 'Piecewise Polynomial Nonlinear Model Reduction', DAC 2003, June 2-6, 2003, Anaheim, California, USA, 2003.

[16] P. D’ALESSANDRO, A. ISIDORI and A. RUBERTI: 'Realization and structure theory of bilinear dynamical systems', SIAM J. Control, 12, pp. 517-535, 1974 
[17] R.R. MOHLER: 'Bilinear Control Processes', New York Academic Press. 1973. 